Mini-Review

Correspondence

Freya Harrison

freya.harrison@zoo.ox.ac.uk

\title{
Microbial ecology of the cystic fibrosis lung
}

\author{
Freya Harrison
}

Department of Zoology, University of Oxford, Oxford OX1 3PS, UK

\begin{abstract}
Understanding the microbial flora of the cystic fibrosis (CF) respiratory tract is of considerable importance, as patient morbidity and death are primarily caused by chronic respiratory infections. However, chronically colonized CF airways represent a surprisingly complex and diverse ecosystem. The precise contributions of different microbes to patient morbidity, and in particular the importance of inter-specific interactions, remain largely unelucidated. The importance of within-species genetic and phenotypic variation has similarly received limited explicit attention. While a host of studies provide data on the microbial species recovered from patients, these are often incomparable due to differences in sampling and data reporting, or do not present the data in a way that aids our understanding of the ecosystem within each patient. This review brings together a cross-section of recent research on the CF airways and the microbes which infect them. The results presented suggest that understanding the CF lung in terms of its community and evolutionary ecology could benefit our understanding of disease progression and influence treatment regimens.
\end{abstract}

\section{Introduction: cystic fibrosis lung communities}

The airways of cystic fibrosis (CF) patients are colonized by pathogenic micro-organisms in infancy, and in the vast majority of cases chronic infections are established. Most patients experience recurrent acute respiratory episodes and eventually die of respiratory failure resulting from infection (Lyczak et al., 2002). Most studies of CF pathogens have focussed on four major bacterial species: Haemophilus influenzae, Staphylococcus aureus, Pseudomonas aeruginosa and Burkholderia cepacia complex (BCC). The prevalence of these species in the airways changes over time, as illustrated in Fig. 1. Most pathogens are acquired from environmental reservoirs, but patient-to-patient transmission also occurs (LiPuma et al., 1990; Salunkhe et al., 2005). The microbiology of the airways can therefore also be influenced by contact between patients (and thus treatment centre isolation policies, attendance of patient support groups, etc.). Colonization by $P$. aeruginosa severely worsens prognosis, promoting more frequent acute exacerbations and a general decline in lung function (Alvarez et al., 2004; Nixon et al., 2001).

Numerous other bacteria invade the CF airways and contribute to morbidity, including Stenotrophomonas maltophilia (shown in Fig. 1), Alcaligenes xylosoxidans and Klebsiella spp. (Lambiase et al., 2006; Lyczak et al., 2002) The range of species reported has widened over the past few decades as life expectancy has increased and detection methods have improved (Rogers et al., 2004). Pathogenic viruses [e.g. respiratory syncytial virus (RSV), adenoviruses, influenza: van Ewijk et al., 2005] and fungi (e.g. Aspergillus and Candida species: Haase et al., 1991) are also common.
In recent years, some CF centres have reported that early, aggressive antibiotic treatment or prophylactic administration of antibiotics can delay or prevent chronic colonization by P. aeruginosa and Staph. aureus (Döring \& Hoiby, 2004; Hoiby et al., 2005; Lebecque et al., 2006). While promising, these initial results are generally based on small samples and treatment does not prevent chronic colonization in all patients. Given the prevalence of CF, small failure rates correspond to a large number of patients for whom alternative therapies must be employed. Further, patients who avoided chronic colonization in these studies still experienced intermittent colonization by the target bacterium, and the incidence of infections by other species was not quantified. Thus the hope of early, aggressive intervention becoming the norm should not lead us to stop trying to understand what happens in the airways when patients do become colonized - especially given the risk of emergent antibiotic resistance.

There exists a wealth of studies of CF lung microflora (see Fig. 2). Unfortunately, our ability to spot patterns in the available data - particularly patterns of coinfection by different species - is hampered by the fact that many surveys do not explicitly report coinfections on a per-patient basis (notable exceptions being Moore et al., 2005; Rogers et al., 2004), do not state patient genotype or disease phenotype (e.g. Burns et al., 1998; Santana et al., 2003), do not record previous antibiotic treatment (e.g. Alvarez et al., 2004) and/ or pool samples from different parts of the respiratory tract (e.g. Nixon et al., 2001). Different surveys use different inclusion criteria and focus on different groups of microbes, with most studies reporting only bacterial pathogens. The 


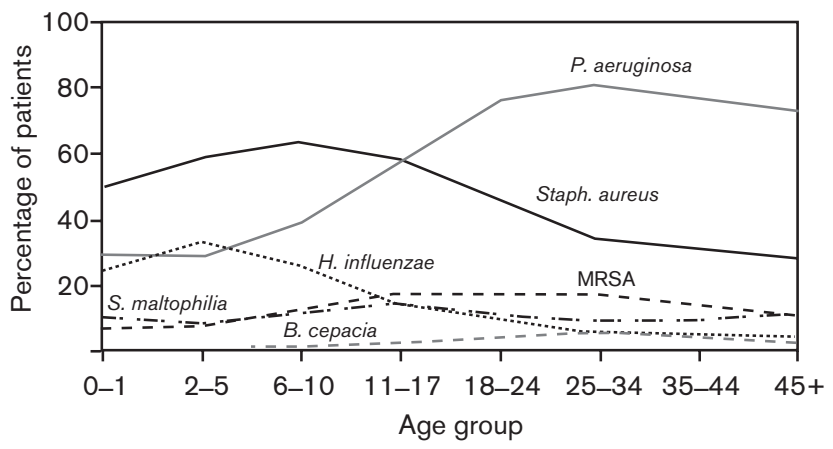

Fig. 1. Prevalence of bacterial respiratory infections by age group in CF subjects. [Reproduced, with modifications, with permission from the Cystic Fibsosis Foundation National Patient Registry (Cystic Fibrosis Foundation, 2004).] MRSA, meticillinresistant Staph. aureus.

picture we have of the lung communities present in $\mathrm{CF}$ patients is, therefore, limited and qualitative.

There are, however, three broad conclusions to be drawn from the published literature. First, coinfections involving different species of bacteria, or bacteria, fungi and viruses, are common and probably the norm. Second, coinfecting species interact, both syngergistically and antagonistically. Finally, pathogen populations within the lung evolve in response to selection pressures exerted by the within-host environment and by other members of the community. These points will now be discussed.

\section{Coinfections and communities}

Several studies explicitly report coinfections of pairs or triplets of bacterial species, while in other cases we can infer from the data given for individual species that a significant proportion of patients were infected by more than one species. Anzaudo et al. (2005) reported that $31 \%$ of patients harboured two or more bacterial species simultaneously, while Hoiby (1974) and Wahab et al. (2004) reported coinfections in 'most patients'. Burns et al. (1998) calculated that they recovered an average of 2.9 species of pathogenic bacteria per sample (range: $0-10$ ), and more recent DNA profiling studies suggest that CF bacterial communities may be even more diverse than suggested by culture-based methods (Rogers et al., 2004). Further, Petersen et al. (1981) found that $13 \%$ of acute respiratory exacerbations among their study population were associated with simultaneous bacterial and non-bacterial infections. The results of a review of reported coinfections are summarized in Fig. 2.

We have, then, evidence for high inter-species diversity in the CF lung. Signficant intra-species diversity is also observed. Burns et al. (1998) recovered, on average, 2.38 phenotypically distinct $P$. aeruginosa isolates per sample per patient (range: 1-6). Further, selection pressures on pathogens are likely to be both temporally and spatially

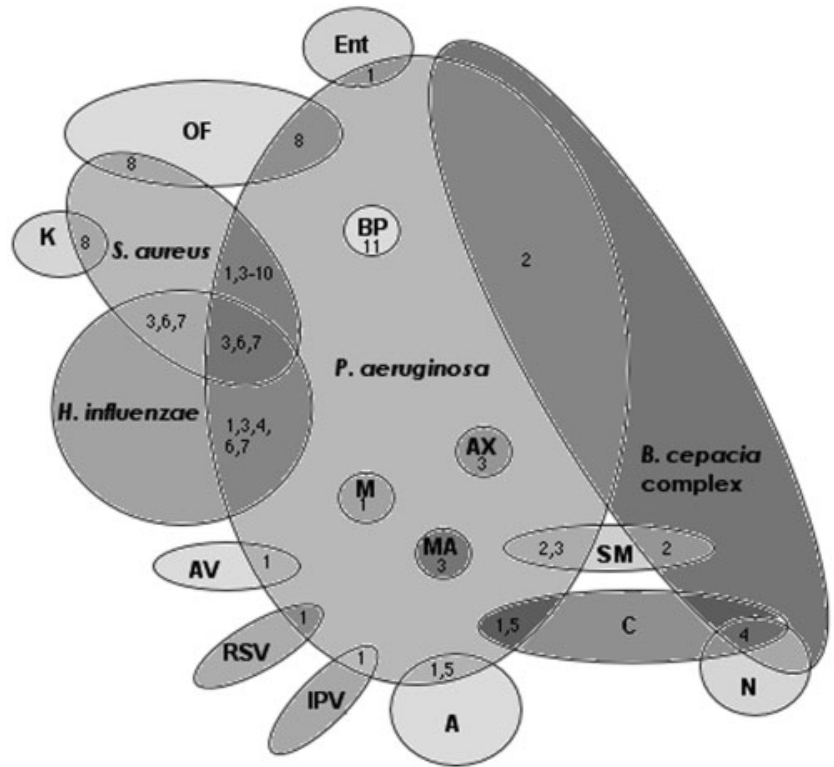

Fig. 2. Venn diagram showing reported coinfections of the CF airways. (NB: coinfection does not necessarily imply direct interaction between species.) A, Aspergillus spp.; AV, adenovirus; $\mathrm{AX}$, A. xylosoxidans; $\mathrm{BP}$, bacteriophage; C, Candida spp.; Ent, enterobacteria; IPV, influenza and/or parainfluenza virus; K, Klebsiella spp.; M, mycoplasma; MA, Mycobacterium abscessus; N, Neisseria spp.; OF, oropharyngeal flora; RSV, respiratory syncytial virus; SM, S. maltophilia. Numbers refer to references: 1, Petersen et al. (1981); 2, Lambiase et al. (2006); 3, Wahab et al. (2004); 4, Moore et al. (2005); 5, Burns et al. (1998); 6, Hoiby (1974); 7, Lording et al. (2006); 8, Santana et al. (2003); 9, Alvarez et al. (2004); 10, Anzaudo et al. (2005); 11, Ojeniyi et al. (1991).

heterogeneous during long-term infections, leading to genetic diversification of founder clones. Smith et al. (2006) genotyped $P$. aeruginosa isolates taken from a single CF patient over a period of 90 months and showed the existence of multiple, related lineages which coexisted for long periods.

Different microbial communities can be found within the same patient. Several authors have noted a poor correlation between species and/or strains present in upper and lower airway communities (Armstrong et al., 1996; Lyczak et al., 2002; Saiman, 2004) and in different lobes of the lung (Gutierrez et al., 2001; Smith et al., 1998). This is unsurprising given the compartmentalized nature of the respiratory system and high viscosity of CF mucus, and means that pooling samples from different lobes (as in Nixon et al., 2001) is unlikely to give a reliable picture of the microbial ecology within a patient.

The outcome of a mixed (multi-strain, multi-genotype or multi-species) infection depends on exactly how coinfecting populations interact. Clinical and laboratory studies have started to elucidate the many ways in which CF pathogens might interact in vivo. 


\section{Community interactions 1: synergism}

Coinfections probably result from sequential, rather than simultaneous, colonization, and it appears that some degree of succession (change in community structure over time) occurs within the CF lung (e.g. Hoiby, 1974; Wahab et al., 2004). Molecular profiling studies such as that of Rogers et al. (2004) provide particularly clear evidence for succession within patients. Succession may depend in part on environmental changes resulting from the action of infecting microbes; alginate production by $P$. aeruginosa, for example, can exacerbate local hypoxia (Worlitzsch et al., 2002), presumably constraining the spectrum of species or strains able to invade. There is also evidence that RSV may render some patients more susceptible to chronic $P$. aeruginosa infection (Petersen et al., 1981).

Historically, synergism in the CF lung has usually been proposed with respect to Staph. aureus 'sensitizing' the lungs to subsequent infection by $P$. aeruginosa (e.g. Burns et al., 1998; but see also Lyczak et al., 2002). However, there is also evidence for antagonistic interactions between these two species (see below). On the other hand, there is very good evidence that $P$. aeruginosa can promote further pathogenesis. Firstly, two endobronchially detectable $P$. aeruginosa quorum-sensing molecules (3-oxo- $\mathrm{C}_{12}$-homoserine lactone and 2-heptyl-3-hydroxy-4(1H)-quinolone) have been shown to possess immunomodulatory effects (Hooi et al., 2004). Secondly, Wahab et al. (2004) reported a possible link between pre-existing, chronic colonization by mucoid $P$. aeruginosa and infection by more rarely observed bacteria such as S. maltophilia, A. xylosoxidans and Mycobacterium abscessus. Moreover, $P$. aeruginosa has been implicated in promoting BCC pathogenesis via the upregulation of BCC virulence factor expression (Riedel et al., 2001).

Synergism can result in increased disease severity in mixedspecies infections. For instance, those patients with coinfections of mucoid $P$. aeruginosa and rare bacterial species observed by Wahab et al. (2004) had significantly worse lung symptoms than did patients colonized by mucoid $P$. aeruginos $a$ alone. The normal oropharyngeal microflora (OF) may similarly exacerbate CF lung disease. In CF patients, avirulent species which are normally confined to the upper airways are frequently recovered from the lower airways (Coenye et al., 2002; Santana et al., 2003). It has been shown (Duan et al., 2003) that the presence of OF exacerbates $P$. aeruginosa-mediated lung damage in infected rats. This resulted from the specific upregulation of $P$. aeruginosa genes linked with pathogenesis, and appeared to be partially attributable to the $P$. aeruginosa cells 'eavesdropping' on intercellular communication between OF constituents (Duan et al., 2003).

Bacteriophage are present at appreciable concentrations in the CF airways (Ojeniyi et al., 1991), and these may affect the pathology of their target bacteria. P. aeruginosa phage recovered from CF sputum have been shown to be capable of inducing changes in $P$. aeruginosa serotype and a shift to polyagglutinability - a trait associated with increased patient mortality (Hoiby \& Rosendal, 1980).

\section{Community interactions 2: antagonism}

Antagonism between organisms within a community is unavoidable due to competition for finite resources. This may involve simple resource competition or direct antagonistic effects on the growth or viability of competitors (interference competition).

There is particularly good evidence for antagonism between $P$. aeruginosa and Staph. aureus. $P$. aeruginosa can lyse the cells of Staph. aureus and other Gram-positive bacteria (Mashburn et al., 2005; Palmer et al., 2005) and it has been demonstrated that CF sputum induces this behaviour, increasing the competitive ability of $P$. aeruginosa in vitro (Palmer et al., 2005). In the case of Staph. aureus, P. aeruginosa can use the iron released by lysis to support its own growth (Mashburn et al., 2005), but the relevance of this within the CF airways is not known.

Two further types of antagonistic interaction have received much attention in the literature on microbial evolution and ecology, but their possible roles in the CF lung have not been explicity addressed. These are bacteriocin production and the breakdown of cooperative behaviours. Bacteriocins are molecules produced by bacteria which kill cells belonging to another clone (Michel-Briand \& Baysse, 2002). Their action is usually limited to conspecific clones, although some bacteriocins play a role in interspecific competition (MichelBriand \& Baysse, 2002). There is a growing body of work on the ecology of bacteriocin production, and it has been shown that bacteriocin-mediated competition can affect virulence. When cells invest resources in bacteriocin production, they reduce their own growth rate as well as that of any sensitive competitors. The result is a reduction in total bacterial density, and hence virulence, in mixed infections (Massey et al., 2004). Bacteriocin production is more common in clinical isolates than in environmental strains (Govan, 1986; Chang et al., 2005), but the extent to which it influences CF lung community structure and virulence has not been addressed. As some authors have suggested therapies based on bacteriocins (e.g. (Michel-Briand \& Baysse, 2002) it may be worthwhile examining the relevance of this behaviour in the CF lung in more detail.

Another type of antagonism is the breakdown of cooperative behaviours due to the evolution of non-cooperative 'cheats'. Microbial populations often increase their growth rate by acting cooperatively - generally by producing 'public goods' such as iron-scavenging siderophores, toxins and degradative enzymes (West et al., 2006). Within a cooperating population, there exists an advantage to becoming a noncooperating 'cheat'; i.e. investing nothing in public good production but utilizing the molecules produced by one's neighbours. If cheats reach sufficiently high frequencies, the effect on resource exploitation and so population density will be significant (Harrison \& Buckling, 2005). Some public 
goods are directly necessary for pathogen virulence, and in such cases cheat-mediated antagonism can decrease virulence (Harrison et al., 2006). Siderophore production by $P$. aeruginosa is a classic example of cooperation (Griffin et al., 2004). However, while necessary in acute infections (Harrison et al., 2006), siderophore production decreases over time in chronic infections (De Vos et al., 2001; Smith et al., 2006). Whether this decline is due to a decreased need for siderophores as more iron becomes available in the CF lung (Stites et al., 1999; but see also Haas et al., 1991), or as a result of increased local competition and/or decreased relatedness (Griffin et al., 2004), remains to be seen. Furthermore, patterns of siderophore production over time can differ markedly between patients (De Vos et al., 2001). If selection pressures differ between patients, it would be useful to determine whether this is attributable to differences in microbial community structure, to differences in the within-host environment, or to founder effects of the colonizing clone. Unfortunately we cannot answer this question with the data currently available. Moreover, the nature of any relationship between siderophore production and CF disease phenotype has not been addressed.

The presence of micro-organisms elsewhere in the host appears to affect the immune response to respiratory infection. In particular, there is evidence that maintaining a healthy gut microflora can antagonize the success of respiratory pathogens. In mice, dietary supplementation with Lactobacillus casei or natural yoghurt has been shown to stimulate the activity of alveolar macrophages, increase clearance of respiratory P. aeruginosa (Alvarez et al., 2001) and expedite recovery from subsequent Streptococcus pneumoniae infection (Villena et al., 2005).

\section{Evolution within the lung}

During chronic infection, pathogens will experience changing selection pressures as they enounter new habitats and different coinfecting species and as they respond to medical intervention. Most of the work on pathogen evolution in the CF lung has focussed on P. aeruginosa. Smith et al. (2006) found evidence of widespread postitive selection across the $P$. aeruginosa genome during 8 years of infection in one patient. The ratio of nonsynonymous to synonymous base changes per site was calculated to be 1.4 and around twothirds of the nonsynonymous mutations were predicted to alter protein function. These authors also reported the in vivo evolution of divergent lineages with different combinations of mutations (mainly single base pair mutations or 1-3 base pair indels). Kresse et al. (2003) also reported adaptation and diversification of this species within the lung, with some novel genotypes enjoying a long-term selective advantage. These authors also found numerous large-scale rearrangements and indels in evolved genotypes.

The most commonly observed phenotypic changes in CF bacterial pathogens over time are antibiotic resistance (Anzaudo et al., 2005; Burns et al., 1998; Lambiase et al.,
2006; Lyczak et al., 2002), mucoidy (Lyczak et al., 2002; Nixon et al., 2001), a loss of cell motility (Mahenthiralingam et al., 1994), the appearance of 'small colony variants' (Haussler et al., 2003; Besier et al., 2007), increased mutation rate (Ciofu et al., 2005) and decreased production of virulence factors associated with acute infections. This last is thought to aid in immune evasion (Smith et al., 2006). The results of Smith et al. (2006) are consistent with these observations, as they recovered numerous loss-of-function (nonsense or frame shift) mutations in genes involved in twitching motility, biofilm formation and the production of compounds required for the establishment of acute infections, as well as mutations that produced a hypermutable phenotype and numerous mutations in multidrug efflux pumps. Efflux pump mutants were shown to have increased resistance to a range of antibiotics.

Some of these evolved traits may be linked with community interactions. For instance, the presence of pilus-binding bacteriophage may select against expression of type IV pili by $P$. aeruginosa and so lead to a loss of twitching motility and biofilm formation.

The increases in both inter- and intra-specific microbial diversity observed during chronic infection (De Vos et al., 2001; Kresse et al., 2003; Smith et al., 2006) could have several possible effects on social interactions. On the one hand, decreases in relatedness could reduce virulence as a result of decreased cooperative host exploitation, or if interference competition maintains a lower total population density. On the other hand, low relatedness could increase virulence if scramble competition favours more voracious clones. The effect of relatedness on virulence has received much discussion, and it has become clear that this relationship depends on the nature of the interactions between coinfecting clones and on the exact behaviours required for host exploitation (West et al., 2006).

\section{How can ecology inform therapy?}

The CF airways represent an evolving ecosystem, with interactions between pathogens affecting disease phenotype. It is therefore advisable to evaluate prophylactic intervention in terms of its community-level effects. For instance, antibiotic treatment to remove particular species will have limited success if other members of the community predispose the patient to recolonization, if treatment simply creates selection pressure for resistance, or if removal of the target pathogen creates an opportunity for other pathogen populations to invade or expand. Additionally, not understanding how populations grow in vivo can mean that in vitro tests of potential therapies produce unreliable results. If we can improve our picture of pathogen ecology within the lung, this will facilitate the development of more promising therapies.

Certain authors (e.g. Moore et al., 2005) have suggested that artificial manipulations of lung communities could form the basis for more effective treatment of chronic infections. 
Treatments based on molecules which block bacterial quorum sensing (Eberl, 2006; Le Berre et al., 2006), bacteriocins (Michel-Briand \& Baysse, 2002), bacteriophage (Toro et al., 2005) and bacteriovorus vibrios (Kadouri \& O’Toole, 2005) have all been suggested. Such interventions could form a prelude to more conventional treatments and increase their efficacy. Some trials of 'ecological medicine' have shown promising results (e.g. Toro et al., 2005). It is clear, however, that a better understanding of the microbial ecology of infections is not only important for predicting their virulence, but is also necessary if we are to develop reliable and safe methods of disrupting these communities. For instance, high doses of pilus-dependent lytic phage administered during the early stages of infection may reduce pathogen population size, but given the potential for some phage to enhance bacterial adaptation and persistence, the details of how specific phage interact with their target bacteria must be elucidated before potentially useful phage can be identified.

Therapies that directly target the airways may not be the only means of increasing resilience to infection. The discovery that a healthy gut microflora protects against respiratory pathogens may prove to be a valuable one. It is especially pertinent as many CF patients receive oral or intravenous antibiotics, whose actions are not localized to the respiratory tract and which could deplete gut microflora. Dietary supplementation with probiotics or prebiotics and/or the preferential use of inhaled antibiotics may be sometimes be advisable and could bolster the success of more direct interventions.

\section{Conclusions: integrating ecology, evolution and medicine}

The microflora of the CF airways commonly consists of numerous multi-species assemblages. Interactions between clones and between species are likely to determine morbidity. Over time, with repeated medical intervention and new colonization events, these communities evolve and their characteristics can change significantly. Our understanding of this ecosystem is sketchy: while we have identified the major pathogenic species and mutants, our knowledge of how these interact within the community is patchy. Understanding the evolutionary ecology of $\mathrm{CF}$ airway infections could help in the search for novel or improved treatments. As it is, the successful removal of one pathogen strain from the lung simply leaves an empty niche which can often rapidly be filled by a new invader (see e.g. Hoiby \& Rosendal, 1980). Given the promising results of early eradication trials, it would also be useful to ascertain how the microbial communities differ in intermittent versus chronic infections. If host factors that affect characteristics of the pathogen community and the course of lung pathogenesis could be identified, there is a chance that future treatments could be optimized to the needs of particular patients.

\section{Acknowledgements}

I would like to thank Mike Brockhurst, Angus Buckling, Andrew Spiers, Howard Jenkinson and two anonymous reviewers for their comments and advice during the preparation of this manuscript and Ruth Massey for bringing interesting publications to my attention.

\section{References}

Alvarez, S., Herrero, C., Bru, E. \& Perdigon, G. (2001). Effect of Lactobacillus casei and yogurt administration on prevention of Pseudomonas aeruginosa infection in young mice. J Food Prot 64, 1768-1774.

Alvarez, A. E., Ribeiro, A. F., Hessel, G., Bertuzzo, C. S. \& Ribeiro, J. D. (2004). Cystic fibrosis at a Brazilian center of excellence: clinical and laboratory characteristics of 104 patients and their association with genotype and disease severity. J Pediatr (Rio J) 80, 371-379.

Anzaudo, M. M., Busquets, N. P., Ronchi, S. \& Mayoral, C. (2005). Microorganismos patógenos aislados en muestras respiratorias de niños con fibrosis quística. Rev Argent Microbiol 37, 129-134.

Armstrong, D. S., Grimwood, K., Carlin, J. B., Carzino, R., Olinsky, A. \& Phelan, P. D. (1996). Bronchoalveolar lavage or oropharyngeal cultures to identify lower respiratory pathogens in infants with cystic fibrosis. Pediatr Pulmonol 21, 267-275.

Besier, S., Smaczny, C., von Mallinckrodt, C., Krahl, A., Ackermann, H., Brade, V. \& Wichelhaus, T. A. (2007). Prevalence and clinical significance of Staphylococcus aureus small colony variants in cystic fibrosis lung disease. J Clin Microbiol 45, 168-172.

Burns, J. L., Emerson, J., Stapp, J. R., Yim, D. L., Krzewinski, J., Louden, L., Ramsey, B. W. \& Clausen, C. R. (1998). Microbiology of sputum from patients at cystic fibrosis centers in the United States. Clin Infect Dis 27, 158-163.

Chang, W., Small, D. A., Toghrol, F. \& Bentley, W. E. (2005). Microarray analysis of Pseudomonas aeruginosa reveals induction of pyocin genes in response to hydrogen peroxide. BMC Genomics 6, 115.

Ciofu, O., Riis, B., Pressler, T., Poulsen, H. E. \& Hoiby, N. (2005). Occurrence of hypermutable Pseudomonas aeruginosa in cystic fibrosis patients is associated with the oxidative stress caused by chronic lung inflammation. Antimicrob Agents Chemother 49, 2276-2282.

Coenye, T., Goris, J., Spilker, T., Vandamme, P. \& LiPuma, J. J. (2002). Characterization of unusual bacteria isolated from respiratory secretions of cystic fibrosis patients and description of Inquilinus limosus gen. nov., sp. nov. J Clin Microbiol 40, 2062-2069.

Cystic Fibrosis Foundation (2004). Patient Registry 2004 Annual Report. Bethesda, MD: Cystic Fibrosis Foundation.

De Vos, D., De Chial, M., Cochez, C., Jansen, S., Tummler, B., Meyer, J. M. \& Cornelis, P. (2001). Study of pyoverdine type and production by Pseudomonas aeruginosa isolated from cystic fibrosis patients: prevalence of type II pyoverdine isolates and accumulation of pyoverdine-negative mutations. Arch Microbiol 175, 384-388.

Döring, G. \& Hoiby, N. (2004). Early intervention and prevention of lung disease in cystic fibrosis: a European consensus. J Cyst Fib 3, 67-91.

Duan, K., Dammel, C., Stein, J., Rabin, H. \& Surette, M. G. (2003). Modulation of Pseudomonas aeruginosa gene expression by host microflora through interspecies communication. Mol Microbiol 50, 1477-1491.

Eberl, L. (2006). Quorum sensing in the genus Burkholderia. Int J Med Microbiol 296, 103-110.

Govan, J. R. (1986). In vivo significance of bacteriocins and bacteriocin receptors. Scand J Infect Dis 49 (Suppl.), 31-37. 
Griffin, A. S., West, S. A. \& Buckling, A. (2004). Cooperation and competition in pathogenic bacteria. Nature 430, 1024-1027.

Gutierrez, J. P., Grimwood, K., Armstrong, D. S., Carlin, J. B., Carzino, R., Olinsky, A., Robertson, C. F. \& Phelan, P. D. (2001). Interlobar differences in bronchoalveolar lavage fluid from children with cystic fibrosis. Eur Respir J 17, 281-286.

Haas, B., Kraut, J., Marks, J., Zanker, S. C. \& Castignetti, D. (1991). Siderophore presence in sputa of cystic fibrosis patients. Infect Immun 59, 3997-4000.

Haase, G., Skopnik, H., Groten, T., Kusenbach, G. \& Posselt, H.-G. (1991). Long-term fungal cultures from sputum of patients with cystic fibrosis. Mycoses 34, 373-376.

Harrison, F. \& Buckling, A. (2005). Hypermutability impedes cooperation in pathogenic bacteria. Curr Biol 15, 1968-1971.

Harrison, F., Browning, L. E., Vos, M. \& Buckling, A. (2006). Cooperation and virulence in acute Pseudomonas aeruginosa infections. BMC Biol 4, 21.

Haussler, S., Ziegler, I., Lottel, A., von Gotz, F., Rohde, M., Wehmhohner, D., Saravanamuthu, S., Tummler, B. \& Steinmetz, I. (2003). Highly adherent small-colony variants of Pseudomonas aeruginosa in cystic fibrosis lung infection. J Med Microbiol 52, 295-301.

Hoiby, N. (1974). Epidemiological investigations of the respiratory tract bacteriology in patients with cystic fibrosis. Acta Pathol Microbiol Scand B Microbiol Immunol 82, 541-550.

Hoiby, N. \& Rosendal, K. (1980). Epidemiology of Pseudomonas aeruginosa infection in patients treated at a cystic fibrosis centre. Acta Pathol Microbiol Scand B Microbiol Immunol 88, 125-131.

Hoiby, N., Frederiksen, B. \& Pressler, T. (2005). Eradication of early Pseudomonas aeruginosa infection. J Cyst Fib 4 (Suppl. 2), 49-54.

Hooi, D. S., Bycroft, B. W., Chhabra, S. R., Williams, P. \& Pritchard, D. I. (2004). Differential immune modulatory activity of Pseudomonas aeruginosa quorum-sensing signal molecules. Infect Immun 72, 6463-6470.

Kadouri, D. \& O'Toole, G. A. (2005). Susceptibility of biofilms to Bdellovibrio bacteriovorus attack. Appl Environ Microbiol 71, 40444051.

Kresse, A. U., Dinesh, S. D., Larbig, K. \& Romling, U. (2003). Impact of large chromosomal inversions on the adaptation and evolution of Pseudomonas aeruginosa chronically colonizing cystic fibrosis lungs. Mol Microbiol 47, 145-158.

Lambiase, A., Raia, V., Pezzo, M., Sepe, A., Carnovale, V. \& Rossano, F. (2006). Microbiology of airway disease in a cohort of patients with cystic fibrosis. BMC Infect Dis 6, 4.

Lebecque, P., Leal, T., Zylberberg, K., Reychler, G., Bossuyt, X. \& Godding, V. (2006). Towards zero prevalence of chronic Pseudomonas aeruginosa infection in children with cystic fibrosis. J Cyst Fib 5, 237-244.

Le Berre, R., Faure, K., Nguyen, S., Pierre, M., Ader, F. \& Guery, B. (2006). Quorum sensing: une nouvelle cible thérapeutique pour Pseudomonas aeruginosa. Med Mal Infect 36, 349-357.

LiPuma, J. J., Dasen, S. E., Nielson, D. W., Stern, R. C. \& Stull, T. L. (1990). Person-to-person transmission of Pseudomonas cepacia between patients with cystic fibrosis. Lancet 336, 1094-1096.

Lording, A., McGaw, J., Dalton, A., Beal, G., Everard, M. \& Taylor, C. J. (2006). Pulmonary infection in mild variant cystic fibrosis: implications for care. J Cyst Fib 5, 101-104.

Lyczak, J. B., Cannon, C. L. \& Pier, G. B. (2002). Lung infections associated with cystic fibrosis. Clin Microbiol Rev 15, 194-222.

Mahenthiralingam, E., Campbell, M. E. \& Speert, D. P. (1994). Nonmotility and phagocytic resistance of Pseudomonas aeruginosa isolates from chronically colonized patients with cystic fibrosis. Infect Immun 62, 596-605.

Mashburn, L. M., Jett, A. M., Akins, D. R. \& Whiteley, M. (2005). Staphylococcus aureus serves as an iron source for Pseudomonas aeruginosa during in vivo coculture. J Bacteriol 187, 554-566.

Massey, R. C., Buckling, A. \& ffrench-Constant, R. (2004). Interference competition and parasite virulence. Proc Biol Sci 271, 785-788.

Michel-Briand, Y. \& Baysse, C. (2002). The pyocins of Pseudomonas aeruginosa. Biochimie 84, 499-510.

Moore, J. E., Shaw, A., Millar, B. C., Downey, D. G., Murphy, P. G. \& Elborn, J. S. (2005). Microbial ecology of the cystic fibrosis lung: does microflora type influence microbial loading? $\mathrm{Br} J$ Biomed Sci 62, 175-178

Nixon, G. M., Armstrong, D. S., Carzino, R., Carlin, J. B., Olinsky, A., Robertson, C. F. \& Grimwood, K. (2001). Clinical outcome after early Pseudomonas aeruginosa infection in cystic fibrosis. $J$ Pediatr 138, 699-704.

Ojeniyi, B., Birch-Andersen, A., Mansa, B., Rosdahl, V. T. \& Hoiby, N. (1991). Morphology of Pseudomonas aeruginosa phages from the sputum of cystic fibrosis patients and from the phage typing set. An electron microscopy study. Acta Pathol Microbiol Immunol Scand 99, 925-930.

Palmer, K. L., Mashburn, L. M., Singh, P. K. \& Whiteley, M. (2005). Cystic fibrosis sputum supports growth and cues key aspects of Pseudomonas aeruginosa physiology. J Bacteriol 187, 5267-5277.

Petersen, N. T., Hoiby, N., Mordhorst, C. H., Lind, K., Flensborg, E. W. \& Bruun, B. (1981). Respiratory infections in cystic fibrosis patients caused by virus, chlamydia and mycoplasma - possible synergism with Pseudomonas aeruginosa. Acta Paediatr Scand 70, 623-628.

Riedel, K., Hentzer, M., Geisenberger, O., Huber, B., Steidle, A., Wu, H., Høiby, N., Givskov, M., Molin, S. \& Eberl, L. (2001). $\mathrm{N}$-Acylhomoserine-lactone-mediated communication between Pseudomonas aeruginosa and Burkholderia cepacia in mixed biofilms. Microbiology 147, 3249-3262.

Rogers, G. B., Carroll, M. P., Serisier, D. J., Hockey, P. M., Jones, G. \& Bruce, K. D. (2004). Characterization of bacterial community diversity in cystic fibrosis lung infections by use of $16 \mathrm{~S}$ ribosomal DNA terminal restriction fragment length polymorphism profiling. $J$ Clin Microbiol 42, 5176-5183.

Saiman, L. (2004). Microbiology of early CF lung disease. Paediatr Respir Rev 5 (Suppl. A), S367-S369.

Salunkhe, P., Smart, C. H., Morgan, J. A., Panagea, S., Walshaw, M. J., Hart, C. A., Geffers, R., Tummler, B. \& Winstanley, C. (2005). A cystic fibrosis epidemic strain of Pseudomonas aeruginosa displays enhanced virulence and antimicrobial resistance. J Bacteriol 187, 4908-4920.

Santana, M. A., Matos, E., do Socorro Fontoura, M., Franco, R., Barreto, D. \& Lemos, A. C. (2003). Prevalence of pathogens in cystic fibrosis patients in Bahia, Brazil. Braz J Infect Dis 7, 69-72.

Smith, D. L., Smith, E. G., Pitt, T. L. \& Stableforth, D. E. (1998) Regional microbiology of the cystic fibrosis lung: a post-mortem study in adults. J Infect 37, 41-43.

Smith, E. E., Buckley, D. G., Wu, Z., Saenphimmachak, C., Hoffman, L. R., D’Argenio, D. A., Miller, S. I., Ramsey, B. W., Speert, D. P. \& other authors (2006). Genetic adaptation by Pseudomonas aeruginosa to the airways of cystic fibrosis patients. Proc Natl Acad Sci U S A 103, 8305-8306.

Stites, S. W., Plautz, M. W., Bailey, K., O’Brien-Ladner, A. R. \& Wesselius, L. J. (1999). Increased concentrations of iron and isoferritins in the lower respiratory tract of patients with stable cystic fibrosis. Am J Respir Crit Care Med 160, 796-801. 
Toro, H., Price, S. B., McKee, A. S., Hoerr, F. J., Krehling, J., Perdue, M. \& Bauermeister, L. (2005). Use of bacteriophages in combination with competitive exclusion to reduce Salmonella from infected chickens. Avian Dis 49, 118-124.

van Ewijk, B. E., van der Zalm, M. M., Wolfs, T. F. W. \& van der Ent, C. K. (2005). Viral respiratory infections in cystic fibrosis. J Cyst Fib 4, 31-36.

Villena, J., Racedo, S., Aguero, G., Bru, E., Medina, M. \& Alvarez, S.

(2005). Lactobacillus casei improves resistance to pneumococcal respiratory infection in malnourished mice. J Nutr 135, 1462-1469.
Wahab, A. A., Janahi, I. A., Marafia, M. M. \& El-Shafie, S. (2004). Microbiological identification in cystic fibrosis patients with CFTR I1234V mutation. J Trop Pediatr 50, 229-233.

West, S. A., Griffin, A. S., Gardner, A. \& Diggle, S. P. (2006). Social evolution theory for microorganisms. Nat Rev Microbiol 4, 597-607.

Worlitzsch, D., Tarran, R., Ulrich, M., Schwab, U., Cekici, A., Meyer, K. C., Birrer, P., Bellon, G., Berger, J. \& other authors (2002). Effects of reduced mucus oxygen concentration in airway Pseudomonas infections of cystic fibrosis patients. J Clin Invest 109, 317-325. 